\title{
Costo-efectividad de dos esquemas de prevención de la infección por citomegalovirus en pacientes con trasplante renal y riesgo intermedio en Colombia
}

\author{
Kateir Contreras¹, María José Vargas¹, Paola García¹, Camilo A. González¹, Patricia Rodríguez", \\ Camilo Castañeda-Cardona ${ }^{2}$, Margarita Otálora-Esteban ${ }^{3}$, Diego Rosselli ${ }^{4}$ \\ 1 Unidad de Nefrología, Servicio de Medicina Interna, Hospital Universitario San Ignacio, Facultad de Medicina, \\ Pontificia Universidad Javeriana, Bogotá, D.C., Colombia \\ 2 Dirección de Proyectos, NeuroEconomix, Bogotá, D.C., Colombia \\ 3 Servicio de Anestesiología, Hospital Universitario San Ignacio, Facultad de Medicina, Pontificia Universidad \\ Javeriana, Bogotá, D.C., Colombia \\ 4 Departamento de Epidemiología Clínica y Bioestadística, Facultad de Medicina, Pontificia Universidad \\ Javeriana, Bogotá, D.C., Colombia
}

Introducción. El citomegalovirus es la causa más frecuente de infección en pacientes con trasplante renal. Existen dos estrategias de similar efectividad para prevenirlo: la profilaxis universal con valganciclovir durante 90 días o el tratamiento anticipado verificando la carga viral semanal y aplicándolo solo si esta es positiva.

Objetivo. Determinar cuál de estas dos estrategias sería más costo-efectiva en pacientes de riesgo intermedio en Colombia.

Materiales y métodos. Se diseñó un árbol de decisiones bajo la perspectiva del tercer pagador considerando únicamente los costos médicos directos en pesos colombianos (COP) del 2014 durante un periodo de un año en una población de pacientes con riesgo intermedio para citomegalovirus (donante positivo y receptor positivo, o donante negativo y receptor positivo). Las probabilidades de transición se extrajeron de los estudios clínicos y se validaron con expertos mediante el método Delphi. Los costos de los procedimientos se basaron en el manual tarifario ISS 2001, con un incremento del $33 \%$ a partir del índice de precios al consumidor (IPC) en salud de 2014, en tanto que los de los medicamentos se extrajeron de las circulares del Ministerio de Salud y del Sistema de Información de Medicamentos (Sismed).

Resultados. La profilaxis universal con valganciclovir resultó ser menos costosa y se asoció con una menor probabilidad de infección. El costo promedio del primer año de tratamiento anticipado sería de COP\$ 30'961.290, mientras que el universal sería de COP\$29'967.834, es decir, un costo 'incremental' de COP\$ 993.456.

Conclusiones. Para los pacientes de riesgo intermedio con trasplante renal en Colombia, la profilaxis universal es la mejor estrategia por ser menos costosa y reducir el riesgo de infección.

Palabras clave: citomegalovirus; costos y análisis de costo; trasplante de riñón; prevención de enfermedades; inmunosupresión; Colombia.

https://doi.org/10.7705/biomedica.v38i0.3613

\section{Cost-effectiveness of two prevention cytomegalovirus infection schemes in renal transplant patients at intermediate risk in Colombia}

Introduction: Cytomegalovirus (CMV) is the most frequent opportunistic infection after renal transplantation. There are two strategies for its prevention: Universal prophylaxis, with valganciclovir for 90 days, and anticipated therapy, using weekly viral load surveillance, and therapy only if positive. Meta-analysis directly comparing both strategies have shown them to have similar effectiveness.

Objective: To determine which strategy is more cost-effective in intermediate risk patients in Colombia. Materials and methods: We designed a third-party payer perspective decision tree, considering only direct medical costs in 2014 Colombian pesos (COP) (USD $\$ 1=$ COP $\$ 2,000)$ and a time horizon of one year. The target population was intermediate $\mathrm{CMV}$ risk patients (positive receptor). Transition probabilities were extracted from clinical studies, validated with a Delphi expert panel method; procedural costs were obtained from the ISS 2001 manual with a 33\% increment based on the Consumer Price Index for 2014, while medication costs were obtained from the official Ministry of Health information system.

\section{Contribución de los autores:}

Kateir Contreras, María José Vargas, Paola García, Camilo A. González y Diego Rosselli: concepción y diseño del estudio Todos los autores participaron en el análisis e interpretación de los datos y en la escritura del manuscrito. 
Results: Universal prophylaxis with valganciclovir was dominant, with lower costs and less probability of infection. The average cost of the first year in anticipated therapy would be COP\$30,961,290, whereas in the case of universal therapy the cost would be COP\$29,967,834 (incremental cost of COP\$ 993,456).

Conclusions: For Colombian renal transplant patients at intermediate risk for CMV infection, universal prophylaxis strategy is the best option.

Key words: Cytomegalovirus; costs and cost analysis; kidney transplantation; disease prevention; immunosuppression; Colombia.

https://doi.org/10.7705/biomedica.v38i0.3613

El trasplante es el tratamiento de elección en pacientes con enfermedad renal crónica avanzada, dado que se asocia con una mejor calidad de vida y una mayor supervivencia que la diálisis (1). La mortalidad global anual en la población de pacientes con trasplante es de 5 a $7 \%$; la primera causa de muerte es cardiovascular (30\%), seguida muy de cerca por las infecciones (21\%) (2). Aunque con los nuevos esquemas inmunosupresores se ha logrado disminuir la incidencia del rechazo, las infecciones siguen siendo uno de los principales factores de morbilidad. La inmunosupresión se asocia con un incremento en el riesgo de infección, por lo cual el cribado y el inicio de la profilaxis son fundamentales (3).

Los costos de las complicaciones infecciosas en trasplantes de órganos sólidos incluyen los de la estancia hospitalaria evitable y el tratamiento, además de la morbilidad y la mortalidad resultantes. En Canadá, la enfermedad por citomegalovirus (CMV) se ha asociado con un aumento de 2,5 veces en los costos del trasplante renal de los pacientes afectados, comparados con aquellos que no presentan la enfermedad (4). La infección viral por CMV es la más delicada después de un trasplante renal; ocurre frecuentemente entre el primero y el cuarto mes, y coincide con las dosis más elevadas del tratamiento inmunosupresor; sin embargo, pasados los seis primeros meses, puede aparecer una infección o una enfermedad tardía, por lo cual el riesgo sigue siendo alto a lo largo del primer año (5).

Entre los factores de riesgo plenamente establecidos para la presencia de alguna infección o enfermedad, el más importante depende de los

\footnotetext{
Correspondencia:

Diego Rosselli, Departamento de Epidemiología Clínica y Bioestadística, Hospital Universitario San Ignacio, Facultad de Medicina, Pontificia Universidad Javeriana, Carrera $7 \mathrm{~N}^{\circ} 40$ - 62, piso 2, Bogotá, D.C., Colombia

Teléfono: (571) 3208320 extensión 2808

diego.rosselli@gmail.com
}

Recibido: 26/09/16; aceptado: 08/05/17 niveles de lgG para CMV, tanto en el donante como en el receptor. Los casos con donante positivo $\left(D^{+}\right)$y receptor negativo $\left(R^{-}\right)$se clasifican como de alto riesgo; cuando el donante y el receptor son positivos $\left(D^{+} / R^{+}\right)$, o el donante es negativo $y$ el receptor positivo $\left(D^{-} / R^{+}\right)$, se clasifican como de riesgo intermedio, y los casos de donante $y$ receptor negativos ( $\left.\mathrm{R}^{-} / \mathrm{R}^{-}\right)$, como de bajo riesgo (6). Otros factores de riesgo mencionados en algunos estudios son los receptores mayores de 60 años de edad, los antecedentes de episodios de rechazo agudo, la infección viral concomitante por polioma, el uso de medicamentos como la ciclosporina, los esquemas con tres medicamentos inmunosupresores comparados con los de dos (7), y, especialmente, la inducción con inmunoglobulina antitimocitos, que aumenta la probabilidad de infección de dos a cinco veces (6).

En Colombia, se han hecho tres estudios descriptivos: en el primero, realizado en Medellín entre 1988 y 1989, se evaluó la seroprevalencia de IgG para CMV en pacientes en lista de espera, donantes cadavéricos, donantes intrafamiliares y en la población general, y se encontraron valores de $92,73,87$ y $74 \%$, respectivamente; se determinó, asimismo, el riesgo para infección por CMV en los pacientes con trasplante, $8 \%$ de los cuales se clasificó como de riesgo alto, $90 \%$ como de riesgo intermedio y $2 \%$ como de riesgo bajo, con un porcentaje de casos de enfermedad por CMV de $9 \%$ (8).

En el segundo estudio, realizado entre 1998 y 2010 tambien en Medellín, se registró $90 \%$ de seroprevalencia en receptores y $90,2 \%$ en donantes, con la siguiente distribución por estatus de riesgo: bajo, 0,8\%, intermedio, $91 \%$, y alto, $8 \%(9)$.

En el último y más reciente estudio descriptivo y retrospectivo realizado entre 2010 y 2014, se evaluó todo el territorio nacional y se documentó una seroprevalencia para CMV de $86,2 \%$ en donantes de trasplantes renales, y de $91 \%$ en receptores, porcentajes similares a los de otros 
países en desarrollo. La distribución del riesgo en ese estudio fue de $7,3,91,4$ y $1,3 \%$ para riesgo alto, intermedio y bajo, respectivamente (10).

La importancia del CMV no radica únicamente en su alta prevalencia e incidencia, sino en la asociación que ha tenido con diversos resultados adversos debidos a la aparición de la enfermedad, o a efectos indirectos asociados con la infección, como el aumento de la incidencia del rechazo del injerto, el factor de riesgo de fibrosis intersticial y la atrofia tubular en los tres meses posteriores al trasplante, la aparición de una glomerulopatía caracterizada por engrosamiento miointimal mediado por quimiocinas codificadas por el CMV (11), así como el aumento del riesgo de infecciones por Candida spp., Pneumocystis jirovecci, Aspergillus spp., virus de Epstein-Barr, herpes virus 6 y 8, y por último, un mayor riesgo cardiovascular y de estenosis de la arteria renal (12).

En ausencia de medidas preventivas, la frecuencia de la infección se ha registrado en 40 a $100 \%$ de los receptores y, la de enfermedad, en cerca de $67 \%(6,13)$. Para combatirlas se han diseñado estrategias de prevención como la profilaxis universal y el tratamiento anticipado (14).

La profilaxis universal implica la administración de medicamentos antivirales a partir de los diez días del trasplante y hasta tres a seis meses después; según los estudios, el ganciclovir y el valganciclovir tienen una efectividad similar (15), aunque se prefiere este último debido a su biodisponibilidad. La dosis recomendada en recientes estudios para pacientes con función renal mayor de $40 \mathrm{ml} /$ minuto, es de $450 \mathrm{mg} /$ día (16). Sin embargo, en años anteriores la dosis sugerida para pacientes con tasa de filtración glomerular mayor de $60 \mathrm{ml} /$ minuto era de $450 \mathrm{mg}$ cada 12 horas. En un metaanálisis reciente se comparó la dosis de $450 \mathrm{mg}$ con la de $900 \mathrm{mg}$ y no se encontraron mejores resultados con mayores dosis (odds ratio, $\mathrm{OR}=1,38: \mathrm{IC}_{95 \%}$ : $0,84-2,25 ; p=0,19$ ), pero la presencia de leucopenia sí se aumentó tres veces $(p=0,0002)(17)$.

El tratamiento anticipado es una estrategia para detectar la replicación en pacientes asintomáticos mediante la determinación semanal de la carga viral con reacción en cadena de la polimerasa (PCR) para CMV, durante los primeros tres meses (18). Para el diagnóstico se ha utilizado la prueba de antigenemia pp65, pero esta no está estandarizada, por lo cual los últimos consensos recomiendan hacer el seguimiento de la carga viral mediante PCR dadas sus características operativas (18).
En algunos metaanálisis recientes de comparaciones directas ha quedado claro que no hay diferencia en cuanto a la presencia de rechazo, la pérdida del injerto, la enfermedad por CMV o la muerte, entre las dos estrategias (19). La profilaxis universal parece asociarse con menores grados de viremia después del trasplante, aunque con desventajas, como una mayor proporción de infecciones tardías y de toxicidad del medicamento (leucopenia, neutropenia y lesión renal) (19). El tratamiento anticipado involucra el gasto semanal de la verificación de la carga viral de CMV mediante PCR y la coordinación de la toma, aunque con la ventaja de que se evita la toxicidad farmacológica (20).

Con respecto al tiempo de duración del tratamiento, en el estudio IMPACT se comparó el tratamiento administrado durante 200 días con el de 100 días en pacientes con riesgo alto $\left(D^{+} / R^{-}\right)$para $C M V$, y se documentó una disminución de la incidencia de la viremia, sin aumento de los efectos adversos, con el tratamiento de 200 días (21). En pacientes con riesgo intermedio para CMV, el tiempo promedio de tratamiento es de 100 días.

En publicaciones previas de comparaciones entre el ganciclovir intravenoso y el valganciclovir en pacientes con riesgo alto $\left(D^{+} / R^{-}\right)$, se evidenció una eficacia similar para prevenir infecciones; sin embargo, a los 12 meses, $36,8 \%$ de los pacientes asignados al tratamiento de 100 días presentó enfermedad tardía comparado con $16,1 \%$ de quienes tomaron el tratamiento durante 200 días. Este beneficio no se replicó en los pacientes con riesgo intermedio (22).

Siguiendo las recomendaciones de las guías y las revisiones previas, se empleó este modelo, con el enfoque de la mejor estrategia frente al riesgo de infección.

En cuanto al uso de timoglobulina, que es uno de los factores de riesgo para CMV, en los estudios consultados se registraron diferentes porcentajes de infección y enfermedad según el medicamento administrado para la inducción. En Grecia, se han reportado datos de infección de 12,8 \% y de enfermedad de 3,9\% (13); en pacientes de riesgo intermedio en Estados Unidos, se ha determinado un porcentaje de infección de 5,7\% con la profilaxis universal y de $55,5 \%$ con el tratamiento anticipado, y $6,3 \%$ de casos de la enfermedad en el primer año (23).

En cuanto a la aparición de otras infecciones bacterianas, por hongos o por otros virus, no se han encontrado diferencias significativas entre las dos estrategias (19). 
Los pacientes de alto riesgo tienen mayor probabilidad de cursar con viremia y enfermedad, por lo tanto, hay consenso en torno a la necesidad de dar profilaxis universal; en los pacientes de bajo riesgo no se recomienda, pero no hay consenso de cuál estrategia utilizar en la población de riesgo intermedio. La frecuencia de infecciones en casos de riesgo intermedio varía en los diversos estudios entre $78 \%$ con tratamiento anticipado y $38 \%$ con profilaxis universal en un estudio (24), y $38,7 \%$ con el tratamiento anticipado y $11 \%$ con la profilaxis universal, en otro (15). El porcentaje de enfermedad fue más parecido y fluctuó entre 6 y $8 \%$. Los efectos secundarios del tratamiento incluyen la leucopenia, presente en algunas series hasta en $22,3 \%$ (25) de los expuestos, la cual se ve potenciada por la interacción con otros fármacos, como el micofenolato de mofetilo, así como por el deterioro de la función renal, la edad avanzada, la hidratación inadecuada y el uso de otros medicamentos nefrotóxicos. La condición de los pacientes suele mejorar entre los tres y los siete días después de suspender el medicamento.

En el estudio sobre un modelo económico en Francia, se encontró que la profilaxis universal era menos costosa, ya que reducía los días de hospitalización y otros cuidados de la salud (4), en tanto que, en las guías colombianas, se aboga por un enfoque estratificado según el riesgo para ajustar el tratamiento (16). En un país como Colombia, con recursos limitados, y en donde los costos son diferentes a los europeos, es importante hacer un análisis de relación entre costos y resultados clínicos, para establecer una guía terapéutica ajustada a nuestras necesidades.

\section{Materiales y métodos}

Con el programa TreeAge Pro Healthcare ${ }^{\circledR}$, versión 2008, se diseñó un árbol de decisiones bajo la perspectiva del tercer pagador (sistema de salud colombiano), considerando únicamente los costos médicos directos durante un año de atención de los pacientes (figura 1). La población incluyó pacientes con trasplante renal de riesgo intermedio para CMV $\left(D^{+} / R^{+}, D^{-} / R^{+}\right)$, y las intervenciones evaluadas fueron la profilaxis universal y el tratamiento anticipado. Además de comparar los costos de las dos alternativas, se compararon otros aspectos como las infecciones, la enfermedad y los eventos adversos evitados.

En el marco de este trabajo, el trasplante renal se consideró de riesgo intermedio para infección por CMV, según los valores serológicos de IgG, cuando el donante y el receptor eran positivos $\left(\mathrm{D}^{+} /\right.$ $\mathrm{R}^{+}$) o el donante era negativo y el receptor positivo $\left(D^{-} / R^{+}\right)$.

La profilaxis universal con valganciclovir era la dosis profiláctica administrada durante 90 días, iniciando el décimo día después del trasplante, dosificada según la tasa de filtración glomerular: si esta es mayor de $40 \mathrm{ml} /$ minuto, se indican 450 mg diarios de valganciclovir, si es de 25 a $35 \mathrm{ml} /$ minuto, 450 mg día de por medio, y si es de 10 a 24 $\mathrm{ml} /$ minuto, $450 \mathrm{mg}$ dos veces a la semana.

El tratamiento anticipado se hizo con un control semanal de CMV durante los primeros tres meses. Si se registran resultados positivos en la carga viral, se inicia la administración de una dosis de $450 \mathrm{mg}$ de valganciclovir cada 12 horas.

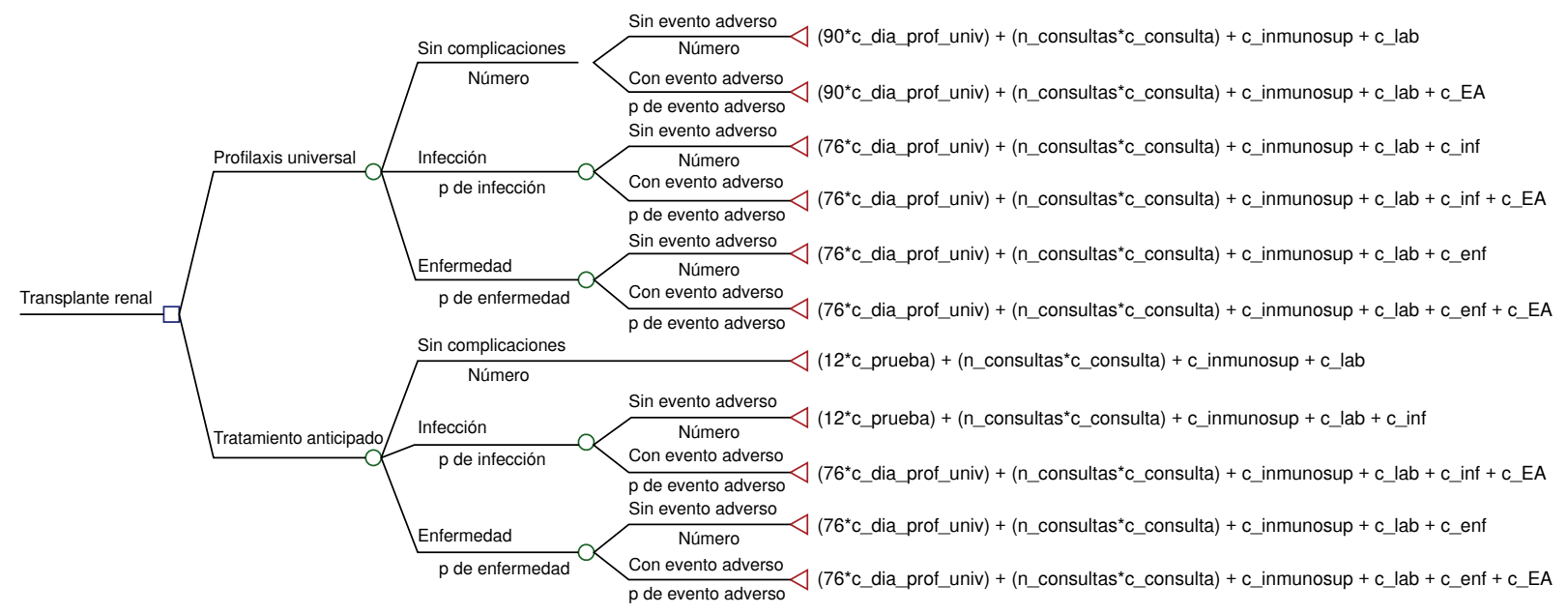

Figura 1. Árbol de decisiones y variables 
Se diagnosticó infección por CMV cuando la PCR era positiva para CMV y el paciente permanecía asintomático (22).

Se diagnosticó enfermedad por CMV cuando la PCR era positiva para CMV y el paciente presentaba síndrome viral con fiebre, malestar, leucopenia o infección invasiva (22).

Se consideró complicación cuando el paciente padecía tanto la infección como la enfermedad por CMV.

Los eventos secundarios considerados fueron leucopenia (menos de 4.000 leucocitos por microlitro) y deterioro renal (aumento de más de $2,5 \mathrm{mg} / \mathrm{dl}$ de la creatinina sérica).

Las probabilidades de transición se extrajeron de los estudios clínicos reportados en revisiones de la literatura científica. Se usó el método Delphi modificado para validar las probabilidades, con la participación de expertos de diferentes partes del país (cuadro 1). La determinación y la medición de los recursos consumidos se hicieron mediante la construcción de un caso de base con datos tomados de los casos de infección y enfermedad por CMV atendidos en una institución especializada en trasplantes, con la información de la cohorte de pacientes del presente estudio y de la revisión de la literatura colombiana disponible.

Los costos se estimaron en pesos colombianos (COP) de 2014. Los costos de los medicamentos con precio regulado se tomaron de las circulares del Ministerio de Salud y, los de los demás, del sistema de información de medicamentos (Sismed), siguiendo las recomendaciones del Instituto de Evaluación de Tecnologías en Salud (IETS) (26). Los costos de los medicamentos se muestran en el cuadro 2.
En cuanto a los costos de los procedimientos, se empleó el manual tarifario ISS 2001 con un incremento de $33 \%$ basado en el índice de precios al consumidor (IPC) en salud del 2014. Los costos de los procedimientos incluidos se presentan en el cuadro 3.

Los estados de salud incorporados al modelo fueron los siguientes: sano, infección, enfermedad, presencia o ausencia de eventos secundarios. No se empleó la tasa de descuento dado el horizonte temporal.

Se hizo un análisis univariado de sensibilidad para cada una de las variables del modelo, empleando intervalos amplios, con el fin de determinar aquellas variables críticas que modificaran los resultados y la conclusión del modelo. Se hizo también un análisis probabilístico del tipo de Monte Carlo, repitiendo las simulaciones mil veces y aplicando distribuciones a cada una de las variables del modelo.

\section{Resultados}

Según nuestras estimaciones, el costo promedio del primer año de un paciente en tratamiento anticipado sería de COP\$ 30'961.290; con profilaxis universal, ese costo sería de COP\$29'967.834 (costo 'incremental' de COP\$ 993.456). Con el tratamiento anticipado se presentarían 48 eventos adversos por cada mil pacientes, mientras que, con el tratamiento universal, se presentarían 124. Por otro lado, por cada mil pacientes con tratamiento anticipado, se presentarían 387 infecciones y, con la profilaxis universal, 110. El número de pacientes con enfermedad sería de 70 por cada mil con cualquiera de las dos alternativas. En total, el número de complicaciones por cada mil pacientes tratados sería de 457 con el tratamiento anticipado y de 180 con la profilaxis universal.

Cuadro 1. Probabilidades de transición empleadas en el modelo

\begin{tabular}{|c|c|c|c|c|}
\hline Probabilidad & $\mathbf{p}$ & Mínimo & Máximo & Fuente \\
\hline Deterioro renal con tratamiento anticipado & 0,0116 & 0,00116 & 0,116 & \\
\hline Deterioro renal con profilaxis universal & 0,03 & 0,003 & 0,3 & \\
\hline Efecto adverso de tratamiento anticipado & 0,105616 & 0,05 & 0,2 & \\
\hline \multicolumn{5}{|l|}{ Efecto adverso de profilaxis universal } \\
\hline Leucopenia con tratamiento anticipado & 0,124 & 0,06 & 0,24 & \\
\hline Leucopenia con profilaxis universal & 0,094 & 0,0094 & 0,4 & \\
\hline Enfermedad con tratamiento anticipado & 0,07 & 0,03 & 0,14 & \\
\hline Enfermedad con profilaxis universal & 0,07 & 0,03 & 0,14 & \\
\hline Infección con profilaxis universal & 0,11 & 0,05 & 0,30 & \\
\hline Riesgo relativo de infección con tratamiento anticipado & 3,52 & 1 & 8 & \\
\hline
\end{tabular}

Fuente: encuesta del método Delphi 
En el análisis univariado de sensibilidad, se encontró que los resultados del modelo en términos de costos se mantenían al modificar las variables de costos o las probabilidades de transición, con excepción de cuatro variables:

Cuadro 2. Costos de los medicamentos

\begin{tabular}{|c|c|c|}
\hline $\begin{array}{l}\text { Costo del tratamiento } \\
\text { por día }\end{array}$ & $\begin{array}{l}\text { Costo } \\
\text { (COP\$) }\end{array}$ & Fuente \\
\hline Micofenolato $(2.000 \mathrm{mg})$ & $\$ 41.075$ & $\begin{array}{c}\text { Circular } 7 \text { de } 2013 \\
\text { del Ministerio } \\
\text { de Salud }\end{array}$ \\
\hline Tacrolimus (6 mg) & $\$ 23.152$ & Sismed \\
\hline Prednisolona (5 mg) & 1.186 & Sismed \\
\hline $\begin{array}{l}\text { Trimetoprim-sulfametoxasol } \\
(160 / 800 \mathrm{mg})\end{array}$ & 47 & Sismed \\
\hline Nistatina $(30 \mathrm{ml})$ & $\$ \quad 1.321$ & Sismed \\
\hline Valganciclovir (450 mg) & $\$ 45.024$ & Sismed \\
\hline $\begin{array}{l}\text { Ganciclovir } \\
\text { ( } 5 \text { mg cada } 12 \text { horas) }\end{array}$ & $\$ 115.735$ & Sismed \\
\hline
\end{tabular}

1) la dosis de valganciclovir, pues si se emplean $900 \mathrm{mg}$ diarios en vez de 450, la profilaxis universal ya no permitiría ahorrar costos;

2) el costo de la verificación de la carga viral de CMV mediante PCR es crítico, y los resultados no favorecerían la profilaxis universal si se redujera el valor actual estimado para la prueba, que es de COP\$ 322.300, a menos de COP\$ 236.000 (una reducción de $27 \%$ );

3) si la probabilidad de enfermedad con la profilaxis universal pasara de la estimación actual de $7 \%$ a $13,4 \%$, o

4) si la probabilidad de infección con el tratamiento anticipado bajara de $38,7 \%$ a apenas $2,9 \%$, pues la profilaxis universal ya no permitiría ahorrar costos (figura 2). En el análisis probabilístico con la simulación de Monte Carlo el costo de la profilaxis universal fue más bajo que el del tratamiento anticipado en $98,2 \%$ de las simulaciones.

Cuadro 3. Costos de procedimientos durante la hospitalización

\begin{tabular}{|c|c|c|c|c|c|}
\hline \multirow{2}{*}{$\begin{array}{l}\text { Procedimiento } \\
\text { Urgencias }\end{array}$} & \multirow{2}{*}{$\begin{array}{c}\text { Frecuencia } \\
\text { de uso }\end{array}$} & \multirow{2}{*}{$\begin{array}{c}\begin{array}{c}\text { Proporción de } \\
\text { pacientes }\end{array} \\
100\end{array}$} & Costo unitario & \multicolumn{2}{|c|}{ Costo ponderado } \\
\hline & & & $\$ 24.193$ & $\$$ & 24.193 \\
\hline Consulta especializada & 1 & 100 & $\$ 22.211$ & $\$$ & 22.211 \\
\hline \multicolumn{6}{|l|}{ Ayudas diagnósticas } \\
\hline Hemograma & 35 & 100 & $\$ 13.659$ & $\$$ & 478.065 \\
\hline Nitrógeno ureico en sangre (BUN) & 35 & 100 & $\$ \quad 4.875$ & $\$$ & 170.625 \\
\hline Creatinina & 35 & 100 & $\$ \quad 4.117$ & $\$$ & 144.095 \\
\hline Carga viral de CMV & 5 & 100 & $\$ 322.300$ & & $1 ' 611.500$ \\
\hline lonograma $(\mathrm{Na}, \mathrm{K}, \mathrm{Cl})$ & 35 & 100 & $\$ 28.941$ & $\$$ & $1^{\prime} 012.935$ \\
\hline Magnesio & 35 & 100 & $\$ 12.211$ & $\$$ & 427.400 \\
\hline Niveles de inmunosupresión & 1 & 100 & $\$ 153.545$ & $\$$ & 153.545 \\
\hline Endoscopia & 1 & 60 & $\$ 302.567$ & $\$$ & 181.540 \\
\hline Colonoscopia & 1 & 60 & $\$ 526.723$ & $\$$ & 316.034 \\
\hline Fibrobroncoscopia & 1 & 5 & $\$ 432.875$ & $\$$ & 21.644 \\
\hline Radiografía de tórax & 1 & 100 & $\$ 27.478$ & $\$$ & 27.478 \\
\hline $\begin{array}{l}\text { Ultrasonografía Doppler del riñón } \\
\text { trasplantado }\end{array}$ & 1 & 100 & $\$ 74.250$ & $\$$ & 74.250 \\
\hline Hemocultivo aerobio & 2 & 30 & $\$ 38.228$ & $\$$ & 22.937 \\
\hline Hemocultivo anaerobio & 1 & 30 & $\$ 51.820$ & $\$$ & 15.546 \\
\hline Coproscópico & 3 & 60 & $\$ 11.112$ & $\$$ & 20.002 \\
\hline Coprocultivo & 1 & 60 & $\$ \quad 8.459$ & $\$$ & 5.075 \\
\hline Tinciones especiales & 3 & 60 & 7.761 & $\$$ & 13.969 \\
\hline \multicolumn{6}{|l|}{ Hospitalización } \\
\hline Consulta de nefrología & 35 & 100 & $\$ 20.077$ & $\$$ & 702.695 \\
\hline Consulta de neumología & 1 & 40 & $\$ 22.211$ & $\$$ & 8.884 \\
\hline Consulta de gastroenterología & 1 & 60 & $\$ 22.211$ & $\$$ & 13.327 \\
\hline Consulta de oftalmología & 1 & 100 & $\$ 22.211$ & $\$$ & 22.211 \\
\hline Consulta de infectología & 1 & 100 & $\$ 22.211$ & $\$$ & 22.211 \\
\hline $\begin{array}{l}\text { Hospitalización general en servicio de alta } \\
\text { complejidad (aislamiento) }\end{array}$ & 35 & 100 & $\$ 136.359$ & $\$$ & 4772.565 \\
\hline \multicolumn{6}{|l|}{ Tratamiento } \\
\hline Valganciclovir & 35 & 90 & $\$ 45.024$ & $\$$ & 1’418.269 \\
\hline Ganciclovir & 10 & 10 & $\$ 115.735$ & $\$$ & 115.735 \\
\hline Total & & & & & $11^{\prime} 818.938,6$ \\
\hline
\end{tabular}




\section{Discusión}

La infección por CMV es frecuente en pacientes con trasplante renal. Los estudios en Colombia han demostrado un aumento en la mortalidad, con un OR de 4,51 (IC $\left.{ }_{95 \%}: 0,94-21,8\right)(27)$; además, está asociada con efectos indirectos, como el aumento de las tasas de rechazo y las bajas tasas de supervivencia del paciente y del injerto $(11,12)$. Las tasas de infección y de enfermedad en pacientes de alto riesgo $\left(D^{+} / R^{-}\right)$son muy altas, por lo que este grupo siempre debe recibir profilaxis universal. En los pacientes de bajo riesgo ( $\mathrm{D} / \mathrm{R}$ ) ), las tasas son bajas, por lo que no se recomienda la profilaxis $y$, en el grupo de pacientes con riesgo intermedio, las tasas descritas en los estudios son variables (40 a $100 \%$, sin tratamiento), por lo que se proponen los dos tipos de estrategias: la profilaxis universal y el tratamiento anticipado (9). Las dos estrategias son igualmente efectivas en términos de infección y enfermedad (3), pero existen escasos estudios a nivel mundial, y en Colombia no hay ninguno, sobre cuál estrategia ofrece mayores ventajas en términos de costos.

En un metaanálisis de estudios en pacientes con trasplante de órganos sólidos y riesgo intermedio, se reportó que el tratamiento preventivo comparado con el placebo fue efectivo para prevenir la enfermedad por CMV (RR=0,29; $\left.I_{95 \%}: 0,11-0,80\right)$ y no se registraron diferencias al compararlo con la profilaxis universal $\left(\mathrm{RR}=0,42 ; \mathrm{IC}_{95 \%}: 0,07-2,65\right)$ (28). En el modelo utilizado en el presente estudio, los pacientes con tratamiento anticipado presentarían mayor número de infecciones comparado con aquellos con profilaxis universal, en tanto que no existirían diferencias en cuanto a los casos de enfermedad.

Si bien la capacidad para disminuir las tasas de infección y de enfermedad ha sido similar con las dos estrategias, preocupa que los eventos adversos, las tasas de resistencia a los antivirales y las infecciones tardías sean mayores con la profilaxis universal que con el tratamiento anticipado. En el metaanálisis citado, no se encontraron diferencias (28).

Al evaluar el tiempo de administración del tratamiento preventivo en el grupo de riesgo intermedio, no se encontró mejoría en la incidencia de la infección y la enfermedad entre una administración de 100 días y una de 200 días; aunque en el estudio IMPACT sí se encontró una diferencia a favor de los 200 días en el grupo de pacientes de alto riesgo (21). Al no existir diferencias aparentes en términos de efectividad, se decidió evaluar si había diferencias en los costos.

El análisis económico evidenció que la profilaxis universal no solo disminuía el riesgo de infecciones, sino que ahorraba costos en comparación con el tratamiento anticipado en pacientes con riesgo intermedio en Colombia. Este resultado se obtuvo en la profilaxis universal con dosis diarias de $450 \mathrm{mg}$ de valganciclovir, y la recomendación es administrarla a pacientes con una tasa de filtración glomerular mayor de $40 \mathrm{ml} /$ minuto, según el consenso colombiano y según el estudio de Kalil, et al. $(16,17)$. Las dosis de $900 \mathrm{mg} /$ día se han asociado con una mayor frecuencia de leucopenia. En el

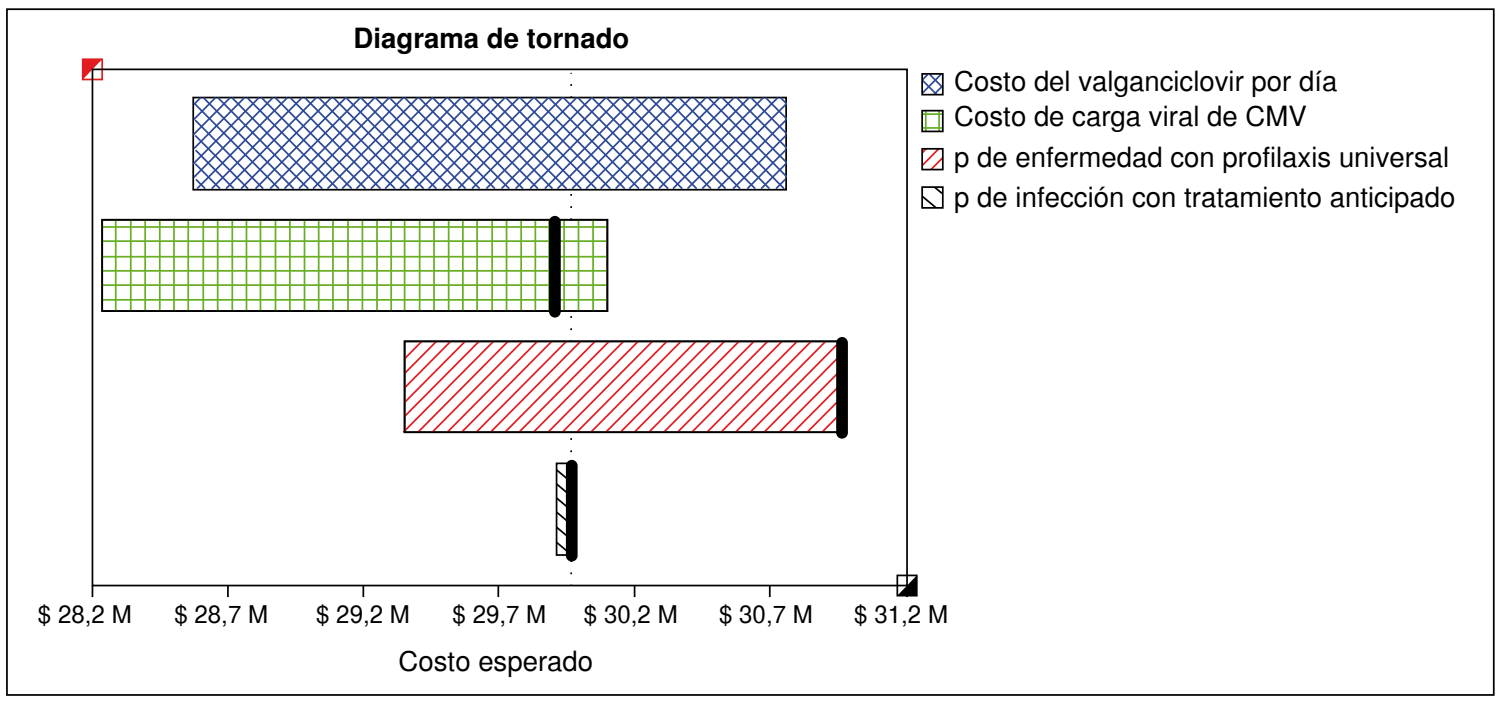

Figura 2. Diagrama de tornado para las cuatro variables de mayor incidencia en las diferencias de costos entre las dos alternativas 
análisis de sensibilidad con una dosis de $900 \mathrm{mg}$, la profilaxis universal dejó de disminuir costos, aunque seguiría siendo costo-efectiva.

La efectividad del tratamiento anticipado se ha demostrado en condiciones ideales, en las cuales se verifica la carga viral mediante PCR cada semana durante los primeros tres meses y, en el momento en que la carga viral se hace positiva, se inicia la administración del fármaco antiviral. En Colombia resulta difícil llevar a cabo este tratamiento debido a las dificultades logísticas para determinar oportunamente la carga viral y las relacionadas con el suministro oportuno del medicamento al paciente, dado que se requiere autorización por no estar contemplado en el plan de beneficios de salud, lo cual hace que los pacientes permanezcan largos períodos sin profilaxis antiviral, con el consecuente aumento del riesgo de infección o de enfermedad y de los efectos en la morbilidad, la supervivencia del injerto, la supervivencia del paciente y los costos.

Dado que el tratamiento con valganciclovir es costoso, era necesario hacer un análisis de costo-efectividad para determinar cuál de las dos estrategias resultaría más conveniente para los pacientes y para el sistema de salud.

Este es el primer estudio en nuestro medio; se espera que contribuya a la práctica diaria de la atención de los pacientes con trasplante renal, aunque tiene las limitaciones propias de los modelos económicos, incluido el empleo de probabilidades de transición y de utilidades derivadas de estudios foráneos.

Si se aceptan sus supuestos y limitaciones, la recomendación sería utilizar la profilaxis universal con valganciclovir durante 100 días en pacientes con riesgo intermedio para $\mathrm{CMV}$, ya que reduciría tanto los costos como los riesgos clínicos de infección y enfermedad por CMV en pacientes con trasplante renal en Colombia.

\section{Agradecimientos}

Al personal de la Unidad de Nefrología y Trasplante Renal del Hospital Universitario San Ignacio.

\section{Conflictos de intereses}

Los autores declaran que no tienen conflictos de intereses relacionados con los contenidos de este artículo.

\section{Financiación}

No hubo fuentes de financiación.

\section{Referencias}

1. Rosselli D, Rueda J, Díaz CE. Cost-effectiveness of kidney transplantation compared with chronic dialysis in end-stage renal disease. Saudi J Kidney Dis Transplant. 2015;26:7338. https://doi.org/10.4103/1319-2442.160175

2. Hernández D, Moreso F. Has patient survival following renal transplantation improved in the era of modern immunosuppression? Nefrologia. 2013;33:171-80. https:// doi.org/10.3265/Nefrologia.pre2012.Nov.11743

3. Lumbreras C. Prevención de las complicaciones infecciosas en el receptor de un trasplante renal. Nefrol Sup Ext. $2011 ; 2: 92-7$

4. Squifflet J-P, Legendre C. The economic value of valacyclovir prophylaxis in transplantation. J Infect Dis. 2002;186(Suppl.1):S116-22. https://doi.org/10.1086/342961

5. Gutiérrez E, Hernández E, Morales E, Praga M. Afectación gastrointestinal severa por CMV tardío: la importancia del tratamiento precoz. Nefrología. 2007;27:779-80.

6. De Keyzer K, van Laecke S, Peeters P, Vanholder R. Human cytomegalovirus and kidney transplantation: $A$ clinician's update. Am J Kidney Dis. 2011;58:118-26. https:// doi.org/10.1053/j.ajkd.2011.04.010

7. Asberg A, Jardine AG, Bignamini AA, Rollag $\mathbf{H}$, Pescovitz MD, Gahlemann CC, et al. Effects of the intensity of immunosuppressive therapy on outcome of treatment for CMV disease in organ transplant recipients. Am J Transplant. 2010;10:1881-8. https://doi.org/10.1111/ j.1600-6143.2010.03114.x

8. Correa M, Ossa J, Builes M, Arbeláez M. Prevalencia de infección por citomegalovirus en receptores y donantes de trasplante renal en Medellín para 1988-1989. Acta Médica Colomb. 1990;15:175-9.

9. Díaz J, Henao J, Rodelo J, García A, Arbeláez M, Jaimes F. Incidence and risk factors for cytomegalovirus disease in a Colombian cohort of kidney transplant recipients. Transplant Proc. 2014;46:160-6. https://doi.org/10.1016/j. transproceed.2013.07.070

10. Arias-Murillo YR, Osorio-Arango K, Cortés JA. Seroprevalencia de citomegalovirus en donantes de órganos y receptores de trasplante renal, Colombia, 2010-2014. Biomédica. 2016;36:24-36. https://doi.org/10.7705/biomedica.v36i0.2938

11. Díaz-Betancur J, Henao JE, Jaimes FA. Efectos de la infección y la enfermedad por citomegalovirus en receptores de trasplante renal. Acta Médica Colomb. 2012;37:131-7.

12. Ramanan P, Razonable RR. Cytomegalovirus infections in solid organ transplantation: A review. Infect Chemother. 2013;45:260-71. https://doi.org/10.3947/ic.2013.45.3.260

13. Giakoustidis D, Antoniadis A, Fouzas I, Sklavos A, Giakoustidis A, Ouzounidis $\mathbf{N}$, et al. Prevalence and clinical impact of cytomegalovirus infection and disease in renal transplantation: Ten years of experience in a single center. Transplant Proc. 2012;44:2715-7. https://doi.org/10. 1016/j.transproceed.2012.09.098

14. Razonable RR. Management strategies for cytomegalovirus infection and disease in solid organ transplant recipients. Infect Dis Clin North Am. 2013;27:317-42. https://doi. org/10.1016/j.idc.2013.02.005 
15. Witzke $\mathbf{O}$, Hauser IA, Bartels M, Wolf $\mathbf{G}$, Wolters $\mathbf{H}$, Nitschke M. Valganciclovir prophylaxis versus preemptive therapy in cytomegalovirus-positive renal allograft recipients: 1-year results of a randomized clinical trial. Transplantation. 2012;93:61-8. https://doi.org/10.1097/TP. 0b013e318238dab3

16. Cortés JA, Yomayusa N, Arias YR, Arroyave IH, Cataño JC, García P, et al. Consenso colombiano para la estratificación, diagnóstico, tratamiento y prevención de la infección por citomegalovirus en pacientes adultos con trasplante renal. Infectio. 2015;20:250-64. https://doi. org/10.1016/j.infect.2015.10.005

17. Kalil AC, Mindru C, Florescu DF. Effectiveness of valganciclovir $900 \mathrm{mg}$ versus $450 \mathrm{mg}$ for cytomegalovirus prophylaxis in transplantation: Direct and indirect treatment comparison meta-analysis. Clin Infect Dis. 2011;52:313-21. https://doi.org/10.1093/cid/ciq143

18. Kotton CN. CMV: Prevention, diagnosis and therapy. Am J Transplant. 2013;13:24-40. https://doi.org/10.1111/ajt.12006

19. Florescu DF, Qiu F, Schmidt CM, Kalil AC. A direct and indirect comparison meta-analysis on the efficacy of cytomegalovirus preventive strategies in solid organ transplant. Clin Infect Dis. 2014;58:785-803. https://doi. org/10.1093/cid/cit945

20. Kalil AC, Levitsky J, Lyden E, Stoner J, Freifeld AG. The efficacy of strategies to prevent organ disease by cytomegalovirus in solid organ transplant recipients. Ann Intern Med. 2005;143:870-80. https://doi.org/10.7326/00034819-143-12-200512200-00005

21. Hellemans $\mathbf{R}$, Beutels $\mathbf{P}$, leven $\mathbf{M}$, Verpooten GA, Bosmans JL. Cost analysis in favor of a combined approach for cytomegalovirus after kidney transplantation: A single-center experience. Transpl Infect Dis. 2013;15:708. https://doi.org/10.1111/tid.12023

22. Kotton CN, Kumar D, Caliendo AM, Åsberg A, Chou S, Danziger-Isakov L, et al. International consensus guidelines on the management of cytomegalovirus in solid organ transplantation. Transplantation. 2013;89:779-95. https://doi. org/10.1097/TP.0b013e31829df29d
23. Luan FL, Kommareddi M, Ojo AO. Universal prophylaxis is cost effective in cytomegalovirus serology-positive kidney transplant patients. Transplantation. 2011;91:237-44. https:// doi.org/10.1097/TP.0b013e318200000c

24. Couzi L, Helou S, Bachelet T, Martin S, Moreau K, Morel D, et al. Preemptive therapy versus valgancyclovir (sic) prophylaxis in cytomegalovirus-positive kidney transplant recipients receiving antithymocyte globulin induction. Transplant Proc. 2012;44:2809-13. https://doi.org/10.1016/j. transproceed.2012.09.029

25. Luna E, Caravaca F, Ferreira F, Fernández N, Martín $\mathbf{P}$, Vargas ML, et al. Effect of cytomegalovirus infection on survival of older kidney transplant patients $(D+/ R+)$ : Impact of valganciclovir prophylaxis versus preemptive therapy. Transplant Proc. 2016;48:2931-37. https://doi.org/10.1016/j. transproceed.2016.06.062

26. Instituto de Evaluación Tecnológica en Salud. Manual para la elaboración de evaluaciones económicas en salud. Bogotá: IETS; 2014. p.36. Fecha de consulta: 27 de abril de 2017. Disponible en: http://www.iets.org.co/ Manuales/Manuales/Manual\%20evaluacio\%CC\%81n\%20 econo\%CC\%81mica\%20web\%2030\%20sep.pdf.

27. López M. Incidencia, frecuencia, etiología y factores de riesgo para la infección en pacientes adultos con trasplante renal de un programa de trasplante de Bogotá (tesis). Bogotá: Universidad Nacional de Colombia; 2015. p. 48. Fecha de consulta: 27 de abril de 2017. Disponible en: http:// www.bdigital.unal.edu.co/49209/1/52965694.2015.pdf.

28. Strippoli GF, Hodson EM, Jones C, Craig JC. Preemptive treatment for cytomegalovirus viremia to prevent cytomegalovirus disease in solid organ transplant recipients. Transplantation. 2006;81:139-45. https://doi.org/10.1097/01. tp.0000183970.71366.da 